\title{
Fatigue damage assessment of uni-directional non-crimp fabric reinforced polyester composite using X-ray computed tomography
}

Jespersen, Kristine Munk; Zangenberg Hansen, Jens ; Lowe, Tristan; Withers, Philip J.; Mikkelsen, Lars Pilgaard

\section{Published in:}

Composites Science and Technology

Link to article, DOI:

10.1016/j.compscitech.2016.10.006

Publication date:

2016

Document Version

Peer reviewed version

Link back to DTU Orbit

Citation $(A P A)$ :

Jespersen, K. M., Zangenberg Hansen, J., Lowe, T., Withers, P. J., \& Mikkelsen, L. P. (2016). Fatigue damage assessment of uni-directional non-crimp fabric reinforced polyester composite using X-ray computed tomography. Composites Science and Technology, 136, 94-103.

https://doi.org/10.1016/j.compscitech.2016.10.006

\section{General rights}

Copyright and moral rights for the publications made accessible in the public portal are retained by the authors and/or other copyright owners and it is a condition of accessing publications that users recognise and abide by the legal requirements associated with these rights.

- Users may download and print one copy of any publication from the public portal for the purpose of private study or research.

- You may not further distribute the material or use it for any profit-making activity or commercial gain

- You may freely distribute the URL identifying the publication in the public portal 


\title{
Fatigue damage assessment of uni-directional non-crimp fabric reinforced polyester composite using x-ray computed tomography
}

Kristine M. Jespersen ${ }^{\mathrm{a}, *}$, Jens Zangenberg ${ }^{\mathrm{b}}$, Tristan Lowe ${ }^{\mathrm{c}}$, Philip J. Withers ${ }^{\mathrm{c}}$, Lars P. Mikkelsen $^{\mathrm{a}}$

${ }^{a}$ Department of Wind Energy, Composites and Materials Mechanics, Technical University of Denmark, DTU Risø Campus, 4000 Roskilde, Denmark

${ }^{b}$ LM Wind Power Blades, Composite Mechanics, Jupitervej 6, 6000 Kolding, Denmark

${ }^{c}$ Manchester X-ray Imaging Facility, School of Materials, University of Manchester, Manchester M13 9PL, United Kingdom

\begin{abstract}
In this study, the progression of tension-tension fatigue $(\mathrm{R}=0.1)$ damage in a unidirectional (UD) composite made from a non-crimp glass fibre fabric used for wind turbine blades is investigated using multi-scale 3D X-ray computed tomography (CT). Initially, a representative volume is examined at one specific damage level. UD fibre fractures are only observed close to the supporting thin transverse backing layers. Furthermore, UD fibre fractures are only observed at locations where backing fibre bundles intersect one another and are at the same time locally close to a UD bundle. In addition, to study the progression of damage as a function of stiffness degradation at higher resolution four samples are subjected to different numbers of cycles before examination by CT. One sample is examined during the initial stiffness drop, two samples during stable stiffness degradation, and one close to final failure. Damage is observed to occur as chains of individual fibre breaks or clusters of fibre fractures rather than large fracture planes. Our work indicates how fracture of UD fibres initiates from intersecting $\pm 80^{\circ}$ backing bundles extending progressively further into the UD layer. The fibre fracture zone becomes more diffuse further from the backing layer. Our work supports
\end{abstract}

\footnotetext{
* Corresponding author. Tel.: +45 28250993.

E-mail address: kmun@dtu.dk (K.M. Jespersen).
} 
a scheme explaining stiffness degradation in terms of UD fibre damage accumulation and demonstrates the importance of 3D and ideally time-lapse imaging studies.

Keywords: A. Polymer Matrix Composites (PMCs), A. Glass fibres, B. Fracture, D. Non-destructive testing, Micro-tomography

\section{Introduction}

With increasing global focus on sustainability, the amount of energy produced from wind turbines has been increasing in recent years [1], and this is expected to continue. However, the challenge of making wind energy cost-competitive relative to fossil fuels remains. Since the power output scales with the swept rotor area (i.e. blade length squared), increasing the blade length decreases the cost of energy of the wind turbine. One of the main challenges when designing long blades is material fatigue. During a wind turbine's life-time of around 20-30 years, it experiences a high number of load cycles (in the range of $10^{8}-10^{9}$ cycles) [2]. The blade is subjected to repeated flapwise bending from the wind and repeated edge-wise bending from the blade weight combined with the rotation [3]. The main load carrying parts of a wind turbine blade consist of uni-directional (UD) glass fibre composite materials made from non-crimp fabrics $(\mathrm{NCF})$. In addition to the fatigue life-time, the stiffness degradation observed during fatigue loading can become a problem in relation to tower clearance. It is therefore of great interest to understand and describe the fatigue damage mechanisms on a micro-structural level for this material, in order to be able to make more fatigue resistant designs and/or to understand how to reduce cost without compromising the fatigue resistance.

\subsection{Fatigue of composites}

The progression of fatigue damage in fibre composites, and how to design against fatigue, has received a great amount of focus in the literature. Particularly within the aerospace industry the initiation of off-axis cracks been the focus of many studies [410]. Fewer studies (e.g. [9-12]) have considered damage progression in terms of fibre 
fractures that occur after the initiation of transverse cracking.

Reifsnider and Jamison [9] examined the fatigue mechanisms in both cross-ply and quasi-isotropic carbon fibre/epoxy composites. In both cases the stiffness degradation during fatigue was observed to include three main stages: a steep initial stiffness reduction stage (stage I), a stable degradation stage (stage II), and an unstable stage with final failure (stage III). They found that for both the cross-ply and quasi-isotropic laminates the initial reduction in stiffness seemed to primarily relate to transverse cracking. The stiffness degradation in stage II was said to decrease stably as a consequence of subcritical element damage such as matrix cracking, matrix splitting along fibre directions, fibre/matrix debonding, and delamination causing the stresses in the laminate to redistribute. The damage would then localise and lead to damage in critical elements such as the $0^{\circ}$ plies. This would then soon be followed by final failure of the laminate $[9,10]$.

Wind turbine blade materials are subjected to a much higher number of load cycles than aerospace and other composite material applications. In addition, because of the specific loading conditions, UD composites are used to carry the main fatigue loads in a wind turbine blade, whereas quasi-isotropic composites are commonly used in the aerospace industry. Therefore, the material design challenges are rather different, because of the need to tolerate UD fibre damage during operation. The UD NCF composites used for wind turbine blades have a bundle structure and experience slightly different damage mechanisms compared to cross-ply and quasi-isotropic composites. However, the shape of the stiffness degradation curve is similar. A previous study by Zangenberg et al. [11] considered tension-tension fatigue damage in a composite material used for wind turbine blades. The study considered a UD NCF glass fibre/polyester composite with thin supporting off-axis backing layers. In their study a fatigue damage evolution scheme was established as discussed below. 


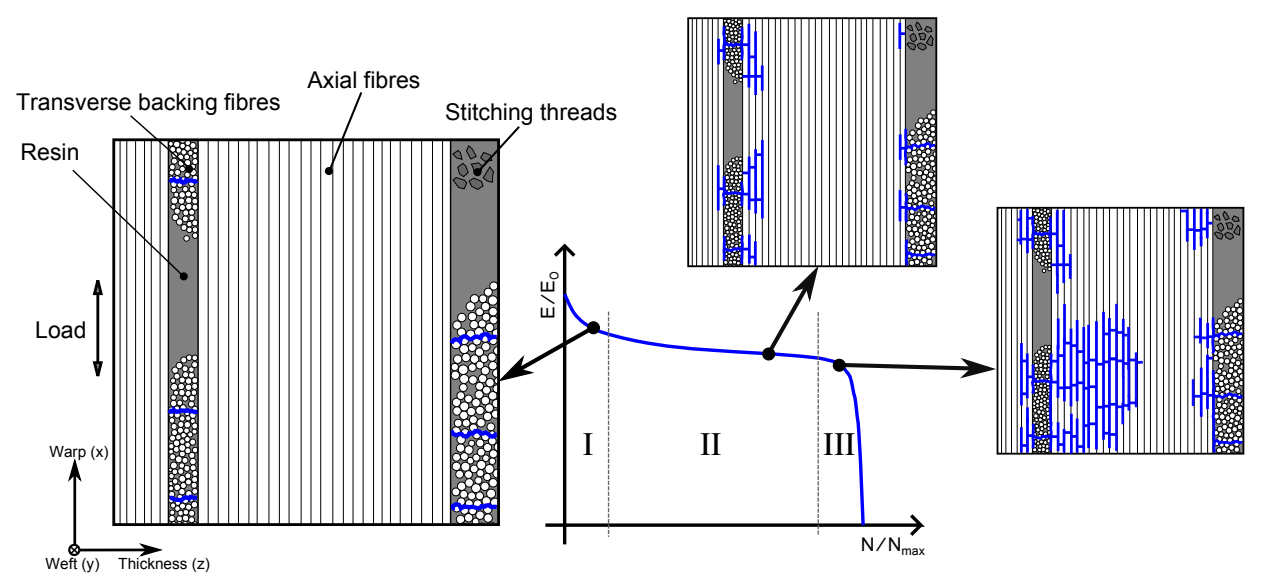

Figure 1: Simplified version of the tension-tension fatigue damage accumulation scheme postulated by Zangenberg et al [11] for a UD composite comprising UD fabric with transverse fibre backing layers.

\subsection{Tension-tension fatigue damage accumulation scheme}

Zangenberg et al. [11] postulated a tension-tension fatigue damage accumulation scheme on the basis of destructive examination techniques (particularly scanning electron microscope (SEM) observations) for the same type of material as considered in the current study (further elaborated later in section 2.1). Fig. 1 shows a simplified version of this scheme (for details see [11]), which also shows the usual shape of the stiffness degradation during fatigue $(R=0.1)$ for this material type. In the scheme, the damage is taken to initiate as transverse cracks in the thin backing layer of criss-crossed bundles of off-axis fibres. These cracks are believed to initially appear in locations where the backing bundles intersect. However, since the initial stiffness drop was found to fit well with the loss of stiffness contributed by the backing layers [11], the transverse cracks are believed to saturate everywhere in the bundles prior to UD fibre damage occurring. After crack saturation the cracks propagate into the UD fibre bundles, causing debonding and fibre fractures. During a stage where the stiffness degrades stably (II in Fig. 1), a steadily increasing number of UD fibre fractures are believed to cause the stiffness degradation. At some point, the number of fibre fractures will have increased the load on the remaining fibres sufficiently to cause rapid static failure. Zangenberg et al [11] also burned off the resin and observed interlaminar failure (longitudinal split- 
ting) penetrating 20-40\% into the UD bundles in the thickness direction. Nevertheless, the destructive nature of their method meant that it was only possible to look at one moment in time, and only to consider 2D surface views, which is partly why the scheme still remains a postulate.

\subsection{X-ray CT of damage in composites}

In recent years, the resolution of non-destructive 3D X-ray computed tomography (CT) has improved significantly. This is opening up a range of new opportunities for the use of X-ray CT for material science purposes [13-15]. In X-ray CT, the sample is placed between an X-ray source and a detector. X-rays emitted from the source pass through the sample and on to the detector, leaving a projection image (as for conventional medical $2 \mathrm{D}$ radiography). The sample is then rotated in steps, for each of which a projection image is acquired. A reconstruction algorithm is then used to reconstruct a 3D image of the considered volume. The contrast in the images is dependent on the difference in material density. Therefore, in relation to composite materials, X-ray CT is particularly well suited for glass fibre/polymer composites due to the relatively large difference in material density (around a factor of 2).

Several recent studies have considered micro-structural damage of composite materials using synchrotron radiation computed tomography. Wright et al. [16, 17] considered static damage in notched carbon fibre reinforced plastic (CFRP) specimens, visualising fibre fractures, intra-laminar delamination and matrix cracking in 3D. Scott et al. [18] performed in-situ static loading of a double notched CFRP specimen, visualising the accumulation of damage for an increasing static load. Synchrotron CT was also used in studies by Garcea et al. [19, 20], to evaluate the fatigue damage evolution of a similar specimen during in-situ start-stop fatigue experiments. All these studies considered samples of a few millimetres in size to obtain high resolution. Synchrotron radiation is particularly suited to short in-situ experiments since the fast scan times makes it possible to capture changes over time. On the downside however, synchrotron facilities are often remotely located, beam time is difficult to access and experiments are typically 
limited to a few days at most, precluding many longer fatigue studies.

The latest laboratory X-ray CT systems can offer micro- and even sub-micron [21] resolution CT comparable to synchrotron systems. These systems make it easier to perform multiple longer timescale studies, albeit using much longer scan times compared to synchrotron CT. Since laboratory X-ray CT systems capable of micro resolution have only recently emerged, only a few studies to date [12, 22-25] have used laboratory systems to study material damage at the micro-structural level. The studies have tended not to consider individual fibre fractures because of insufficient resolution (e.g. [22]). As for synchrotron radiation CT, studies of fibre fractures have examined sample sizes of only a few millimetres (e.g. [12]). Some studies have used staining techniques to increase the visibility of the damage thereby lessening the resolution requirements. In a recent study by $\mathrm{Yu}$ et al. [23], the fatigue damage progression of matrix cracks and delaminations in a larger test specimen (16x2mm in cross-section) was studied for a 3D woven carbon fibre composite at 10.7 micron voxel size by staining with contrast agent. For that case the contrast agent was found to properly penetrate into material [24]. However, in order for staining to be effective, all the damage must be interconnected to the outer surfaces. This might not be the case for the UD NCF used for wind turbine blades, since the off axis layers are thin compared to the UD layer thickness and there are resin rich regions, which are large compared to the backing bundles. Furthermore, the damage at the surface is likely to be caused by edge effects and the focus of the current study is on what is happening a distance away from the edges.

The new advances within laboratory X-ray CT make it possible to move from destructive $2 \mathrm{D}$ testing to visualising damage in $3 \mathrm{D}$ and by extension over time. In this paper, we study the axial tension-tension fatigue damage progression in a UD glass fibre composite in 3D. Based on qualitative observations, the proposed damage accumulation scheme [11] is evaluated with a focus on the three dimensional arrangement of the damage features. Initially, the general locations of fatigue damage regions are investigated using a field of view (FoV) large enough to contain several regions of the backing where the fibre bundles intersect, however with limited resolution. Based on 
the knowledge obtained on the nature of the damage regions, scans have then been performed at higher resolution on a systematic set of four samples each subjected to a different number of fatigue load cycles.

\section{Material and methods}

\subsection{Composite material system}

The composite material is a UD glass fibre/polyester composite made from a noncrimp fabric. The composite consists of layers of UD fabric stacked on top of each other and infused with resin using the vacuum assisted resin transfer moulding (VARTM) infusion technique. For each layer of fabric, parallel UD fibre bundles are stitched to supporting off-axis backing bundles, giving it a fabric-like structure. The stitching thread is made from polyester, hence it has the same density as the matrix material and will not be visible in the performed X-ray CT scans. The dry fabric is shown in Fig. 2, which also illustrates the stitching pattern used to stitch the UD and backing bundles together. The backing bundles are present to keep the UD bundles in place, and do not have a significant effect on the material axial stiffness and strength properties, though they do slightly contribute to the transverse stiffness.

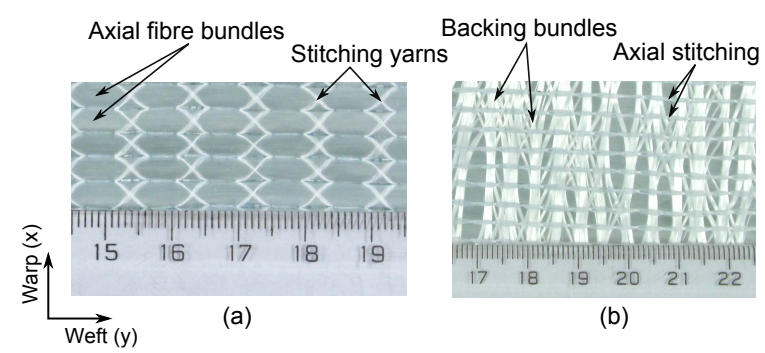

Figure 2: Photographs of UD1322 UD fabric viewed from (a) the UD bundle side and (b) the backing side.

Fig. 3 shows a 3D rendering of the fibre architecture obtained by laboratory Xray $\mathrm{CT}$. It is seen that the backing bundles are quite unevenly spaced as a result of the stitching pattern, and intersect in some locations. In this paper, the term "backing layer" denotes the space between the layers of parallel UD bundles in the final composite, 
as also indicated in Fig. 4. These layers contain both backing bundles and resin-rich regions as indicated in Fig. 3.

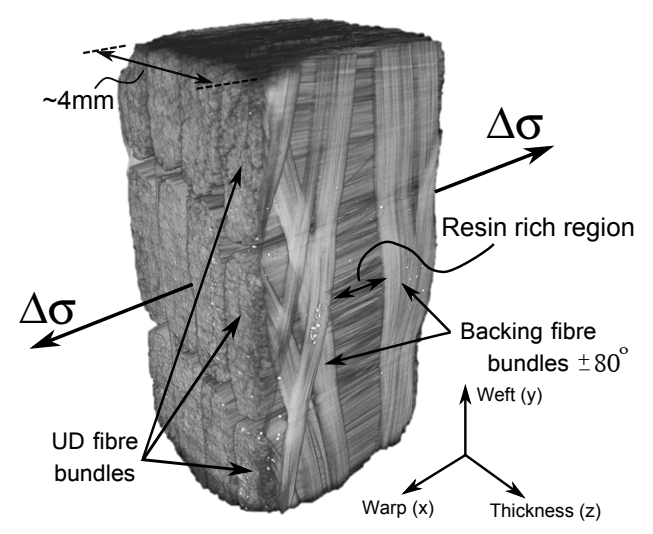

Figure 3: 3D tomograph of the glass fibre/polyester composite micro-structure obtained by X-ray CT (9.7 $\mu \mathrm{m}$ voxel). The matrix has been rendered transparent by thresholding.

The composite layup has 4 layers of UD fabric in the stacking sequence $[\mathrm{b} / 0, \mathrm{~b} / 0]_{s}$ where "b/0" symbolises a layer of fabric with " $b "$ denoting the $\left( \pm 80^{\circ}\right)$ backing layer and " $0 "$ the axial $\left(0^{\circ}\right)$ UD layer of the fabric. Fig. 4 shows a schematic of the stacking sequence and the fabric specification is summarised in Table 1. The matrix is a proprietary unsaturated polyester. It has been cured for more than 24 hours at room temperature followed by a post curing sequence at $40^{\circ} \mathrm{C}$ in an oven for more than 16 hours. This is a curing sequence resulting in a fully cured laminate.

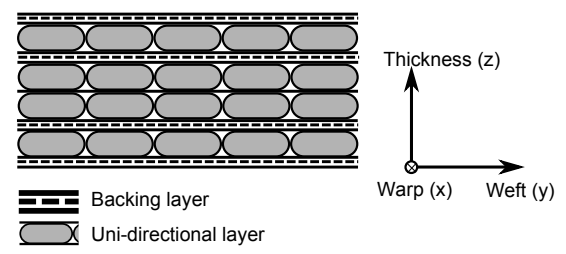

Figure 4: Schematic showing the composite stacking sequence. The UD fibre direction is out of the plane.

Fig. 5 shows a 3D rendering of a higher resolution laboratory CT scan where the matrix material has again been rendered invisible. The approximate dimensions of the UD and backing layers in the final composite are indicated in the figure, which also 
Table 1: Fabric specification (UD1322)

\begin{tabular}{lllll}
\hline Category & Unit & Axial fibres & Backing fibres & Stitching thread \\
\hline Material & - & $\mathrm{H}+$ glass & E-glass & Polyester \\
Area density & {$\left[\mathrm{g} / \mathrm{m}^{2}\right]$} & 1322 & 60 & 15 \\
Linear density & {$[\mathrm{tex}]$} & 2400 & 68 & 7.6 \\
Avg. filament dia. & {$[\mu \mathrm{m}]$} & 17 or 24 & 9 & $\mathrm{~N} / \mathrm{A}$ \\
\hline
\end{tabular}

shows that the backing layers are considerably thinner than the UD layers. In addition, the thickness of the backing layer varies quite a lot as the backing bundles lie on top of one another and cross-over in some locations, whereas in other locations there are no fibres at all.

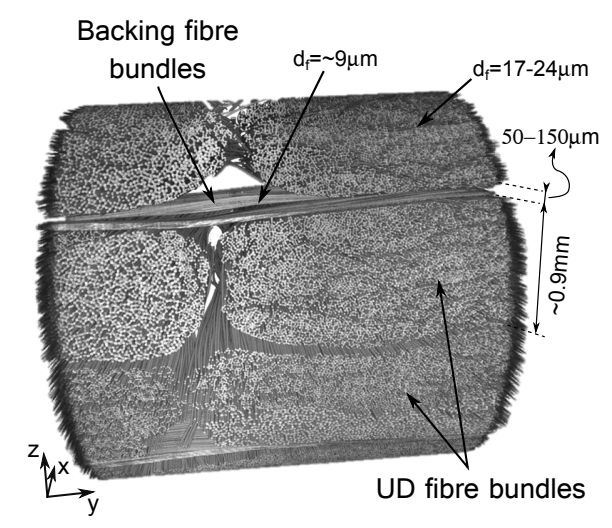

Figure 5: 3D reconstructed image recorded with the MR settings in Table 2 (3.37 $\mu m$ voxel size). The matrix and stitching has been rendered invisible to show the fibre architecture.

\subsection{Fatigue testing}

Two rectangular plates of the composite outlined in section 2.1 were manufactured and cut into butterfly shaped fatigue test specimens as shown in Fig. 6. The butterfly geometry is a special optimized geometry for testing UD composite materials [26], as standard plane specimen geometries tend to fail in the grip area instead of the gauge area. It is reported [26] to give longer fatigue life-times than the standard plane ISO specimens (ISO 527) [27]. The tests were performed at a Servo hydraulic 


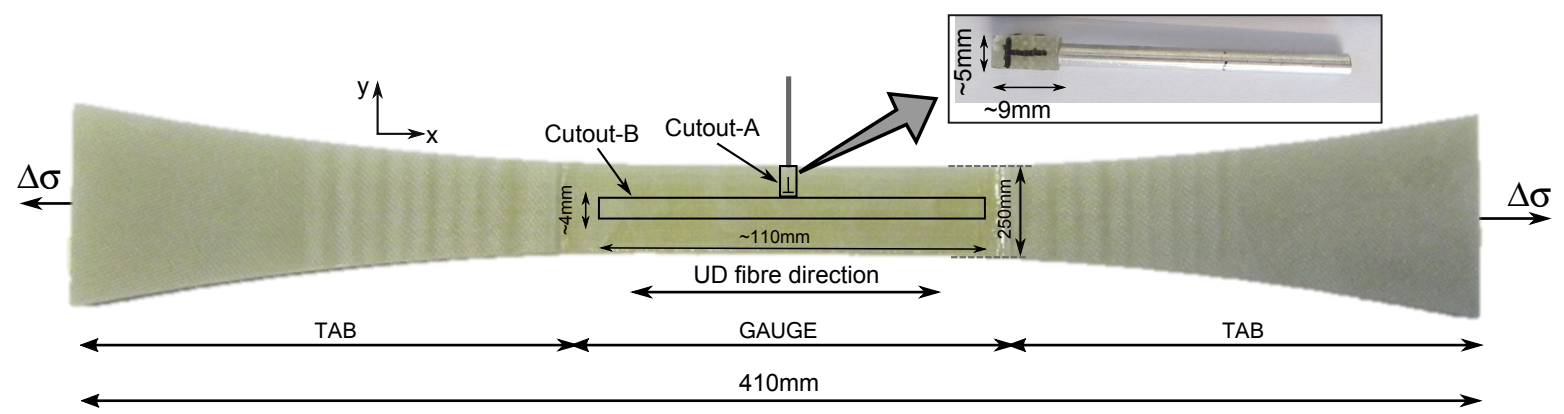

Figure 6: Butterfly specimen geometry (full length of $410 \mathrm{~mm}$ ) showing approximate cut-out locations and dimensions. The photo in the top-right corner shows the cut-out A sample glued to an aluminium pin for easy mounting in the scanner.

Instron test machine under load control with a sinusoidal waveform. The stress ratio was $\mathrm{R}=0.1$, the frequency $5 \mathrm{~Hz}$ and the strain was monitored by two extensometers $([25 \mathrm{~mm} / \pm 2.5 \mathrm{~mm}])$. The maximum fatigue load was $30 \%$ of the static strength of the composite. At this stress level the composite is damaged progressively during fatigue testing as also discussed by Talreja [28].

To examine the damage at different stages during the fatigue life several tests were interrupted prior to failure. A total of 9 tests were carried out and are listed below.

- Two samples were interrupted at 2,000 cycles (during the initial stiffness drop region marked by "I" in Fig. 1).

- Two samples were interrupted at 55,000 cycles (during the stable stiffness degradation regime marked by "II" in Fig. 1).

- One sample was interrupted at 85,000 cycles (during the stable stiffness degradation regime marked by "II" in Fig. 1).

- One sample was interrupted at 95,000 cycles (where the stiffness starts to drop drastically marked by "III" in Fig. 1).

- Three samples were tested to failure. 


\subsection{X-ray computed tomography}

All the samples prepared for X-ray CT in this study were cut using a diamond blade cutter, and two types of cut-out geometries were used (See Fig. 6). The cutoutA geometry was glued to an aluminium pin, which makes it simple to repeatedly mount the sample in the scanner in the same way, even if taken out of the holder. For the cutout-B geometry it is possible to perform further fatigue testing on the down sized sample, which is of interest for future time-lapse studies.

The X-ray CT scans were carried out on a Zeiss Xradia Versa 520 scanner with a 2000x2000 pixel detector and a bit depth of 16. Two different sets of scan settings labelled "MR" (medium resolution) and "HR" (high resolution) were used (see Table 2). Data visualisation and segmentation was done using the commercial software "Avizo 9.0" from FEI, and image reconstruction was done using the software "XMReconstructor - Cone Beam 10" from Zeiss.

The MR scan settings considers a larger FoV than HR, and is performed with a binning of 2 , which means that the X-ray counts from $2 \times 2$ pixels on the detector are combined giving a higher image intensity making it possible to decrease the exposure time (see [29] for more detailed information on X-ray CT). This means that the total scan time is shorter, however, at the compromise of resolution since the detector resolution is binned from e.g. 2000x2000 to $1000 \times 1000$ pixels. Nonetheless, it is much more challenging to process the data from a binning 1 scan, since the file-size is much larger (see Table 2). However, when looking for small features compromising the resolution over scan time and file-size might result in loss of important detail.

An overview of the scans performed in this study can be found in Table 3, and the purpose of these scans is explained in the following sections. The datasets MR3, HR2, HR3, and HR4 along with videos related to Fig. 9 and 11 can be found online [30]. ${ }^{1}$

\footnotetext{
${ }^{1}$ The datasets for MR3 and HR2-4 along with videos of ROI from Fig. 9 and 11 are available at: http://doi.org/10.5281/zenodo.154714 [30]. The data sets can be loaded using the open source software "ImageJ". For use of these data, please make a reference to this paper.
} 
Table 2: Versa $520 \mathrm{X}$-ray CT imaging conditions

\begin{tabular}{|c|c|c|}
\hline Category & MR & $\mathrm{HR}$ \\
\hline Optical & $3.98(4 X)$ & $3.98(4 X)$ \\
\hline \multicolumn{3}{|l|}{ Magnification } \\
\hline $\begin{array}{l}\text { Source to sample } \\
\text { distance }\end{array}$ & $15 \mathrm{~mm}$ & $11 \mathrm{~mm}$ \\
\hline $\begin{array}{l}\text { Detector to sample } \\
\text { distance }\end{array}$ & $15 \mathrm{~mm}$ & $20 \mathrm{~mm}$ \\
\hline Exposure time & $3 \mathrm{~s}$ & $6 \mathrm{~s}$ \\
\hline No. of projections & 3201 & 3201 \\
\hline Accelerating Voltage & $80 \mathrm{keV}$ & $60 \mathrm{keV}$ \\
\hline Binning of pixels & 2 & 1 \\
\hline Pixel size & $3.37 \mu \mathrm{m}$ & $1.2 \mu \mathrm{m}$ \\
\hline $\begin{array}{l}\text { Field of view on } \\
\text { detector }\end{array}$ & $3370 \mu \mathrm{m}$ & $2400 \mu \mathrm{m}$ \\
\hline $\begin{array}{l}\text { Avg. reconstructed } \\
\text { file size }\end{array}$ & $2 \mathrm{~GB}$ & $16 \mathrm{~GB}$ \\
\hline
\end{tabular}

\subsubsection{Medium resolution scans: Locating damage regions}

In X-ray CT, the resolution decreases with increasing size of the FoV, which means that a small FoV is necessary to obtain high resolution. In previous studies $[11,26]$ the UD fibre fracture regions were found to be local phenomena and therefore locating these regions can be difficult if the FoV is too small. To identify where the damage regions typically occur, a cut-out of $\sim 4 \times 5 \times 10 \mathrm{~mm}$ was initially studied with a voxel size of $3.37 \mu \mathrm{m}$ and cylindrical FoV of $\sim 3.4 \mathrm{~mm}$ in diameter and $\sim 3.4 \mathrm{~mm}$ in height ("MR3" in Table 3).

The scanned sample was cut-out from the test specimen subjected to 85,000 cycles where damage is expected to be present. The FoV of the scans encompassed almost the full width of the sample, but excluded the backing layers at the sample surface 
Table 3: Performed X-ray CT scans. The datasets MR3, HR2, HR3, and HR4 can be found online [30].

\begin{tabular}{lllll}
\hline Sample & Dataset & Cycles & Geom. & Note \\
\hline S3 & MR3 & 85,000 & Cutout-A & 3 overlapping scans along length \\
S1 & HR1 & 2,000 & Cutout-B & Initial stiffness drop regime \\
S2 & HR2 & 55,000 & Cutout-B & Stable stiffness degradation regime \\
S3 & HR3 & 85,000 & Cutout-A & Stable stiffness degradation regime \\
S4 & HR4 & 95,000 & Cutout-B & Damage localisation \\
\hline
\end{tabular}

in the thickness direction. Three overlapping scans were performed along the sample length covering a total length of $\sim 6.2 \mathrm{~mm}$. This was done in order to include several regions prone to damage in the scanned volume. The scan settings were chosen with a FoV judged to be as large as possible, while still being able to see fibre fractures. Because of the limited resolution, however, segmentation of individual fibre fractures would include a considerable amount of uncertainty. Therefore higher resolution scans were subsequently performed when studying the damage regions in detail.

\subsubsection{High resolution scans: damage regions in several samples}

To study the damage at different stages of the fatigue life, the samples subjected to 2,000, 55,000, 85,000 and 95,000 cycles were examined at high resolution. The related scan settings and cut-out geometry used for these scans were previously outlined in Table 3. The scans were performed with a pixel size of $1.2 \mu \mathrm{m}$ and a cylindrical FoV of $\sim 2.4 \mathrm{~mm}$ diameter $\mathrm{x} 2.4 \mathrm{~mm}$ height. By utilizing the knowledge of the location of the damage regions obtained from the MR scan, it was possible to locate regions prone to damage despite the small FoV by examining the 2D projection images when setting up the scan. 


\section{Results and discussion}

\subsection{Fatigue tests}

The S-N curve for the composite is shown in Fig. 7, which also shows the tests that were stopped before failure for X-ray CT inspection. It should be noted that the fitted curve is based on only 5 points and normally a minimum of 10 points is required for a well defined curve. Fig. 8 shows the stiffness degradation curves for all the fatigue tests performed at $\epsilon / \epsilon_{0}=0.3$, where $\epsilon_{0}$ is the static strain to failure, and highlights the curves for the four test specimens examined using X-ray CT. In Fig. 8 it is seen that the initial stiffness drop is similar for all the tests, but there is some scatter in the gradient in stage II. For the considered strain level, $\epsilon / \epsilon_{0}=0.3$, only three tests in total were continued all the way to failure and the number of cycles to failure are seen to vary considerably. It is a known problem that the lifetime from tests of this UD composite can vary as much as by a decade. In part this is due to the fact that some of the samples fail outside the gauge region - even when using the butterfly geometry [26]. Since the slope of the curves is generally similar (particularly for the four highlighted tests), it makes more sense to focus on the percentage stiffness drop relative to the absolute number of load cycles and not the percentage of total life.

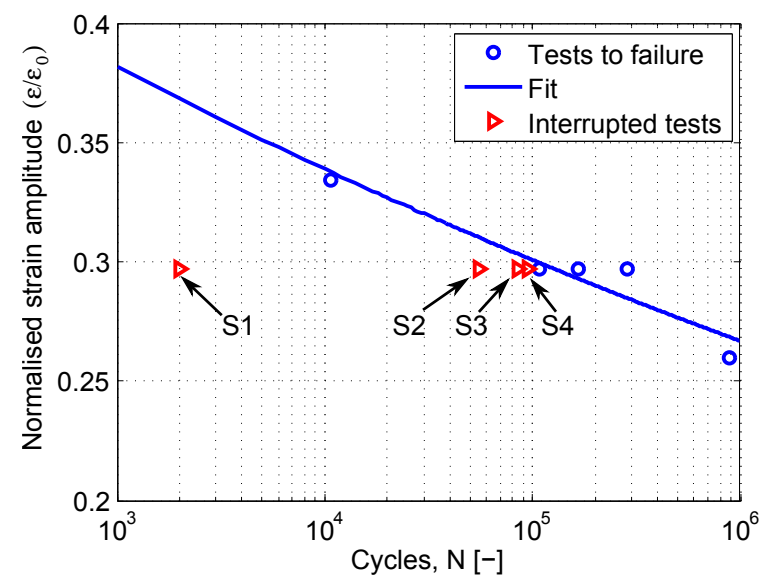

Figure 7: S-N curve including the interrupted tests S1-4. 


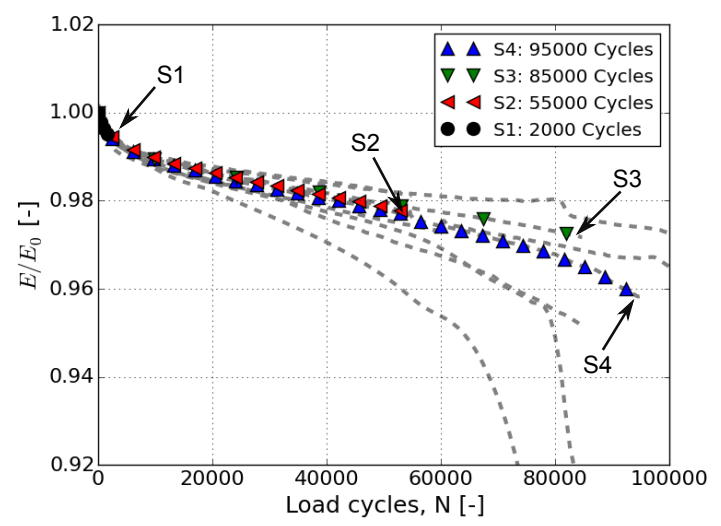

Figure 8: The degradation in stiffness occurring at an imposed strain amplitude $\epsilon / \epsilon_{0}=0.3$ during tension fatigue testing. The symbols denote the stiffness degradation curves for the interrupted test samples imaged by X-ray CT at high resolution. The dashed lines show all the tests. The numbers of cycles at which the tests (S1-S4) were interrupted are marked by arrows.

\subsection{Location of damage regions}

Fig. 9 shows a stitched $2 \mathrm{D}$ view of the three medium resolution scans making up the MR3 dataset. Three regions of interest (ROI) contained load carrying UD fibre fractures and were located locally close to the thin off-axis backing layers (also previously discussed in [31]). For ROI 1 and ROI 3 in Fig. 9, damage was observed on both sides of the backing. For ROI 2, however, damage was only observed on the side to which the backing bundles were stitched. However at this location a layer of matrix was present in between the backing and the UD bundles. Videos slicing through the ROIs in the $\mathrm{x}-\mathrm{y}$ plane along with the full data sets can be found online [30]. Based on the observations of this volume, damage was found to be present only where both of the following criteria are met (see also Fig. 10):

- there is no visible distance between the backing and the UD bundles.

- the backing bundles intersect.

Similar mechanisms where damage initiate at bundle cross-over regions has also previously been observed for ceramic matrix composites (e.g. [32, 33]). 


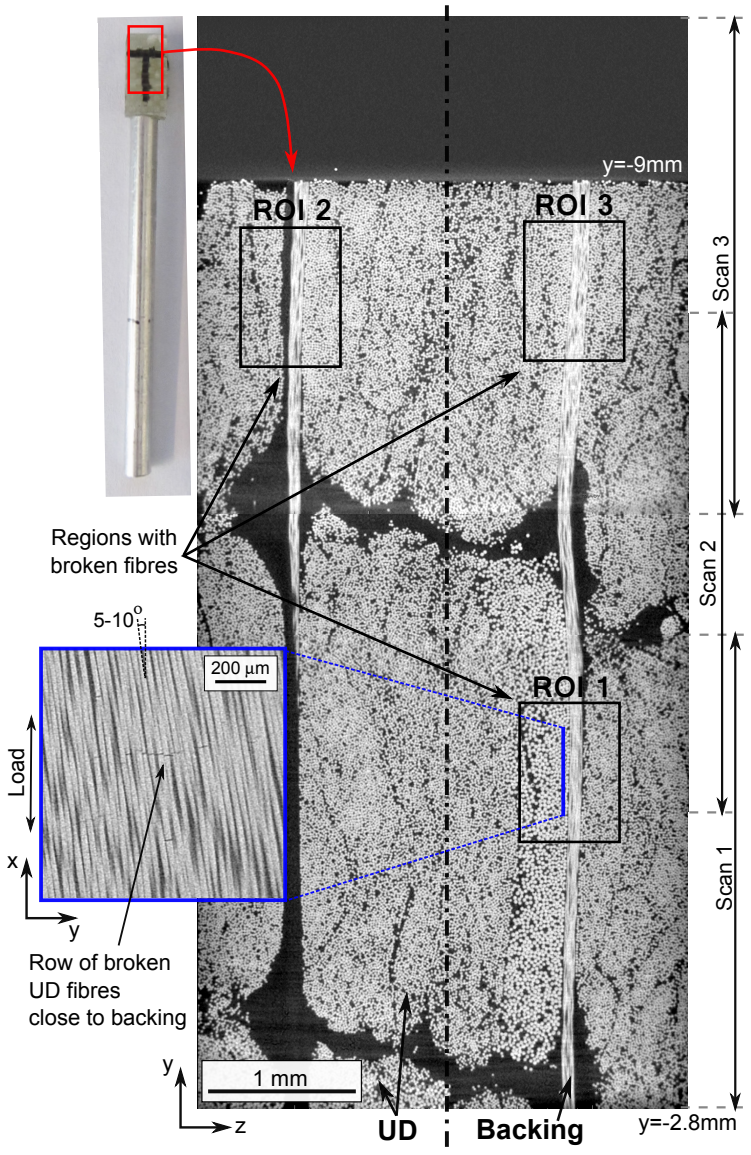

Figure 9: Stitched virtual interior slice comprising the three CT scans that make up the scan region for the MR3 dataset. The ROI's marked are the only locations where damage was observed. The inset shows broken UD fibres close to the backing layer.

Fig. 9 also shows an example of the observed broken fibres, and in this location the UD fibres are seen to be misaligned $\left(5-10^{\circ}\right)$ to the loading direction. However, this was not the case for the other damage regions and therefore, as discussed previously [31], it is not believed have much influence. The main damage characteristics are listed below.

- UD fibre fractures are typically observed as a row of fibre breaks likely emanating from transverse cracking in the matrix of the nearby backing fibres.

- The fibre fracture zone spreads out further from the backing layer, and after 85,000 cycles, no damage was observed beyond approximately 300 microns from the backing. 


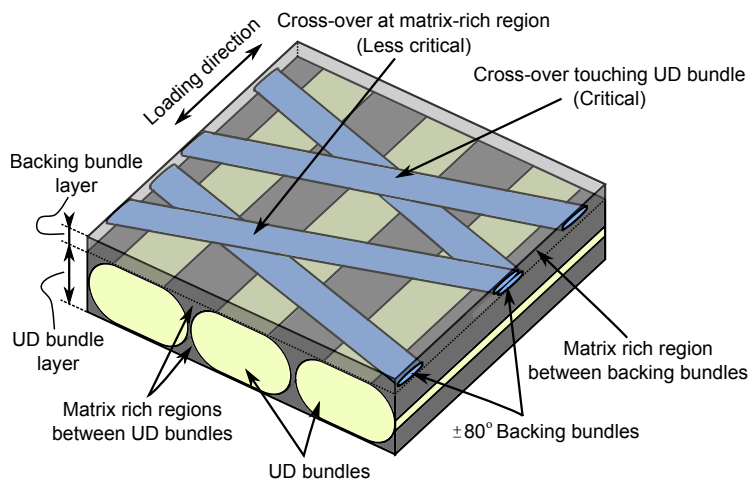

Figure 10: Simplified sketch of one layer of fabric in the composite showing the difference between critical and less critical cross-over regions. The critical cross-over regions are where both $\pm 80^{\circ}$ and a UD bundle cross over and touch each other.

\subsection{Observed damage at different stages of life}

For the scans performed at high resolution ("HR" in Table 2), it was possible to see some additional damage features compared to the medium resolution scan (MR3). These additional damage features were previously outlined in [34], and include:

1. Matrix/interface cracks parallel to the UD fibres.

2. Matrix cracks extending through the backing bundles into the matrix rich regions between the UD bundle.

3. Matrix cracks in backing bundles (observed rarely).

Of the above mentioned damage features, 1 and 2 are not believed to significantly influence the stiffness degradation, as only a few broken UD fibres were observed near these damage features. Matrix cracks in the backing (point 3) however, are believed to be the cause of the initial stiffness drop observed in the fatigue tests. In addition, they are believed to be the initiator of UD fibre fractures and therefore are of considerable interest. In some locations however, the resolution was not sufficient to detect matrix cracks in the backing bundles as the existing transverse cracks most likely became closed with the load removed.

Fig. 11 shows a virtual slice taken from the high resolution ROI scan performed on the cutout-A sample (HR3). A transverse matrix crack in the backing spans the entire 
backing layer, and broken UD fibres are observed locally near the matrix crack. The matrix crack is seen to span two layers of backing at this location. A video showing all the slices through the full volume of Fig. 11 can be found online [30].

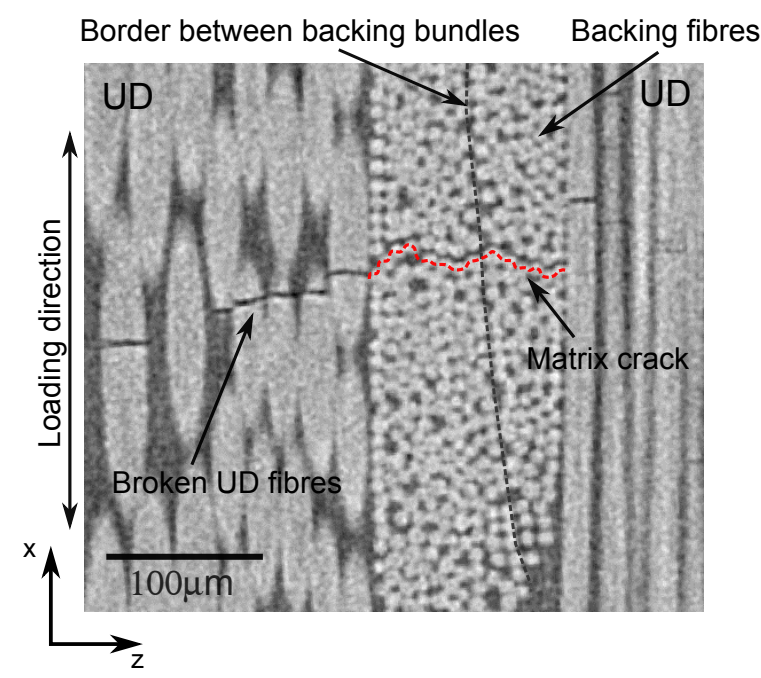

Figure 11: Virtual slice showing a matrix crack through two intersecting backing bundles and adjacent UD fibre fractures (HR3 scan). A video slicing through the volume can be found online [30]

\subsubsection{Damage during initial stiffness drop, stage I}

For the initial stiffness drop sample (S1), only subjected to 2,000 cycles, it was not possible to identify any damage in the scans performed at $1.2 \mu \mathrm{m}$ voxel resolution. It is believed however, that matrix cracks are present in the backing layers at this stage, and that they cause the small initial stiffness drop as discussed in section 1.2. It is possible that the crack opening is small due to a small amount of damage in the sample. Further studies are ongoing in order to confirm the presence and locations of matrix cracks at this stage e.g. by applying static tension to the sample during CT scanning to keep open any cracks.

\subsubsection{Damage while stiffness degrades stably, stage II}

For the sample subjected to 55,000 cycles (S2) only one small damage region was found in the observed volume ( $\sim 2.4 \mathrm{~mm}$ diameter $\mathrm{x} 2.4 \mathrm{~mm}$ height). The broken UD fibres were segmented manually using the Avizo software. The distribution of fibre 
fractures (represented by red discs) in the UD bundles is shown in Fig 12. As was also seen in section 3.2, the damage in Fig. 12 occurs where the backing bundles intersect and there is no visible distance between the UD and backing bundles. The damage region is quite small, but it is possible that larger damage regions are present at other locations in the sample because the FoV associated with the scans only cover a small portion of the test sample.

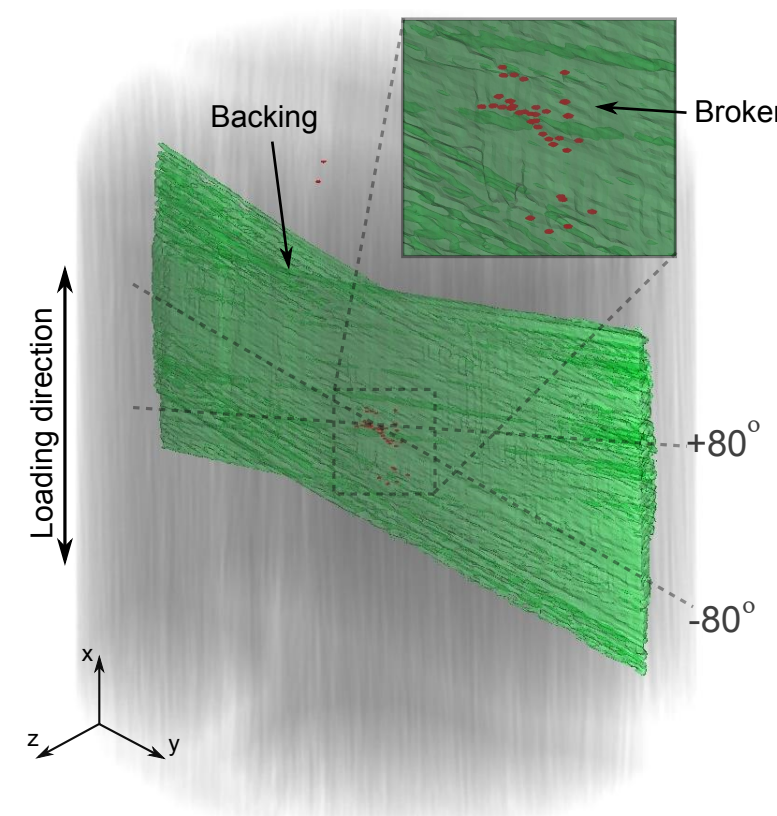

(a) 3D view

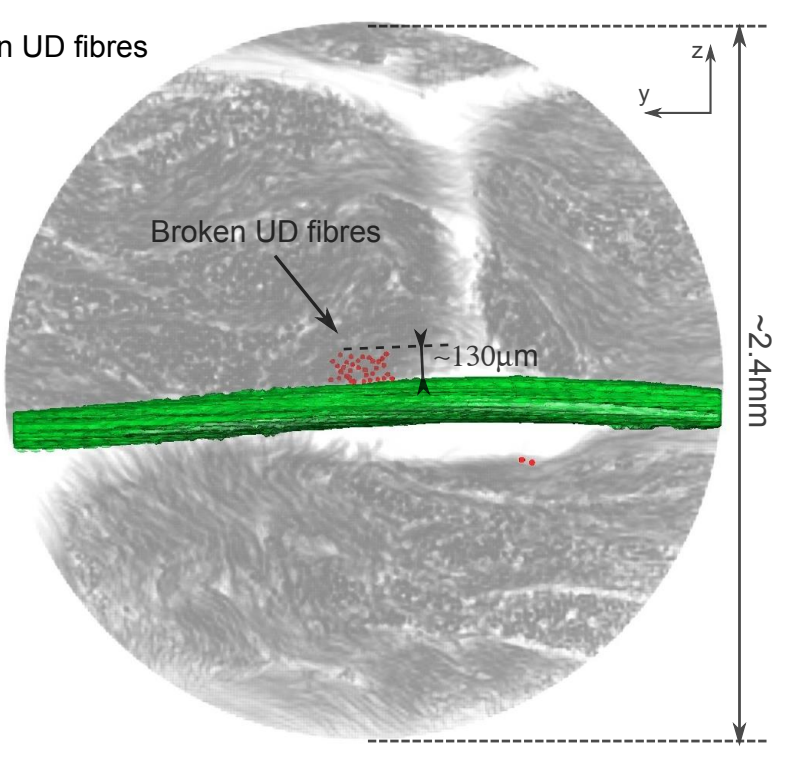

(b) Top view

Figure 12: a) 3D visualisation and b) top view of fibre fractures for the high resolution scan of S2 (dataset HR2). The fibre fractures are marked as red discs, the thin off-axis backing bundles are marked in green, and the UD fibres have been rendered transparent for clarification.

Similarly, Fig. 13 shows the segmentation of individual UD fibre fractures for the high resolution scan (HR3) performed on the test specimen subjected to 85,000 cycles (at ROI 1 in Fig. 9). It is evident that the fibre fractures next to the backing are aligned with the backing fibre direction (for more cases see also [31]), which could be an indication of damage initiation from a matrix crack as suggested by Fig. 11. A bit further from the backing, the UD fibre fractures are more dispersed forming a damaged volume where the fibre damage does not necessarily follow the direction of the backing 
fibres. Within this volume, both single chains of fibre fractures, small clusters, and small planes of fibre fractures were observed. The chains of single fibre breaks are not necessarily straight nor entirely perpendicular to the load direction. From Fig. 13 it is also clear that the damage mechanism is a local 3D phenomenon, and considering this problem in 2D may give misleading conclusions.

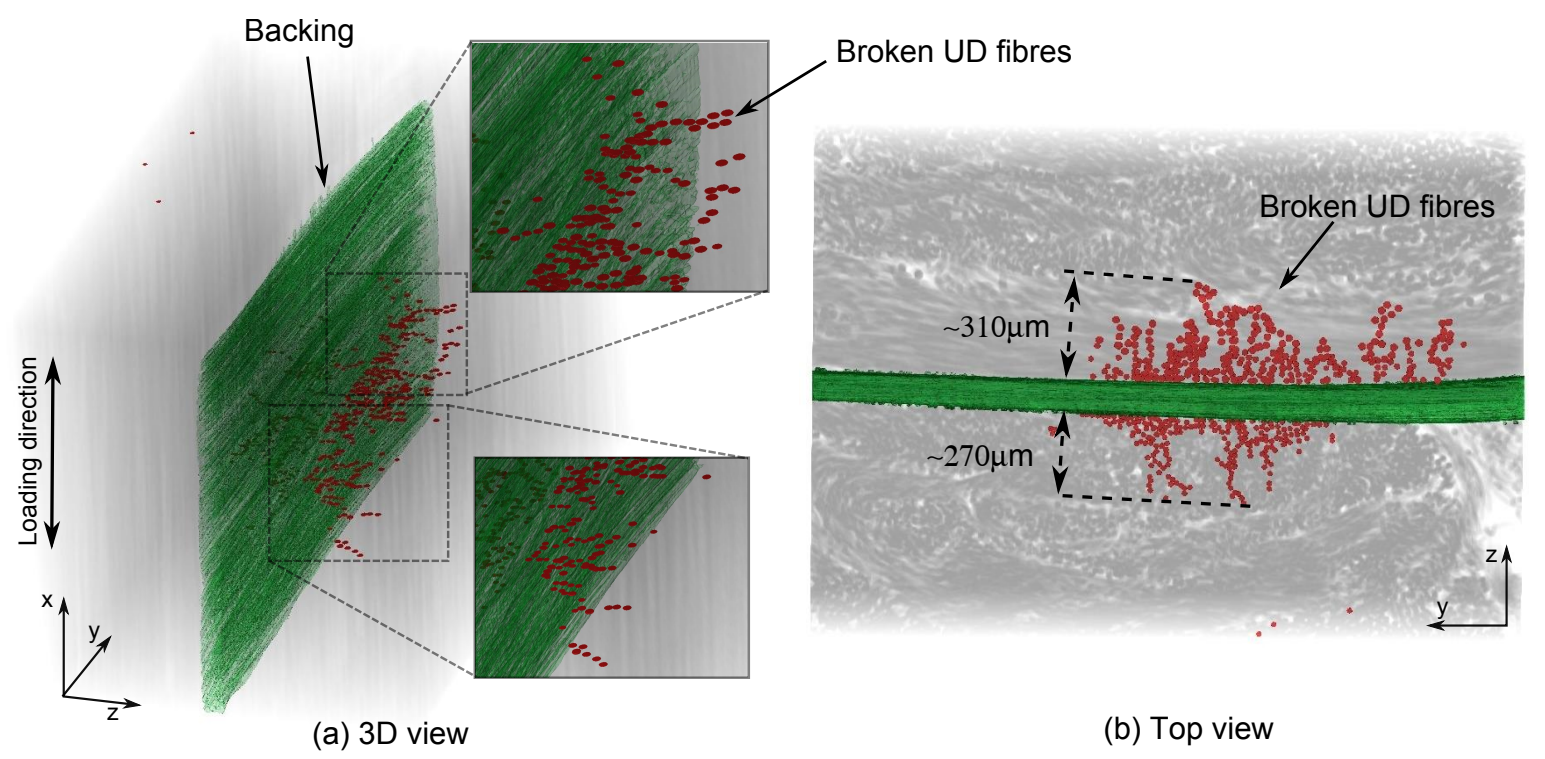

Figure 13: a) 3D visualisation and b) top view of fibre fractures for the high resolution scan of S3 (dataset HR3). The fibre fractures are marked as red discs, the thin off-axis backing bundles are marked in green, and the UD fibres have been rendered transparent for clarification.

\subsubsection{Damage as stiffness starts to fall drastically, stage III}

At the onset of drastic damage (S4) a larger damage region is observed (Fig. 14). As observed for S2 and S3, the fibre fractures closest to the backing are seen to line up with the orientation of the backing fibres (see Fig. 15). As also observed for S2 and S3, the UD fibre fractures are spread out into a damaged zone further away from the backing. In this region, the fractures seem to form chains and clusters rather than fracture planes, whereas the damage in Fig. 13 appeared to be arranged in planes to a higher extent. From the top view in Fig. 14, it can be seen that the damage zone extends less than halfway into the UD bundle $(\sim 370 \mu \mathrm{m})$, while from the $3 \mathrm{D}$ view it 
is seen that it extends quite far $(\sim 2 \mathrm{~mm})$ along the UD fibre direction. This was not observed as significantly for the other loading stages.

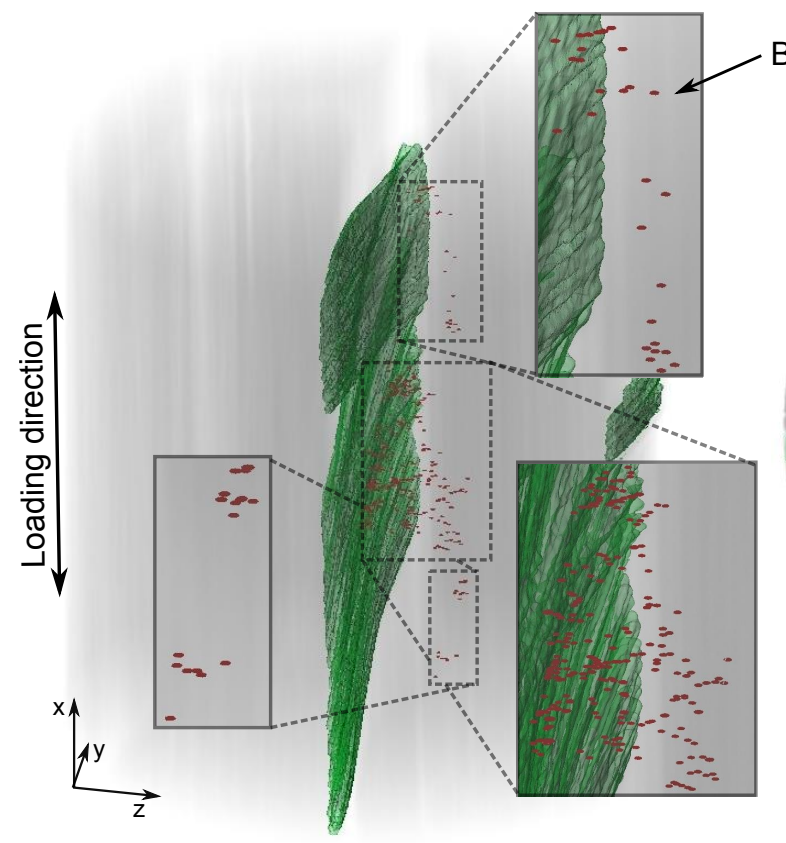

(a) 3D view

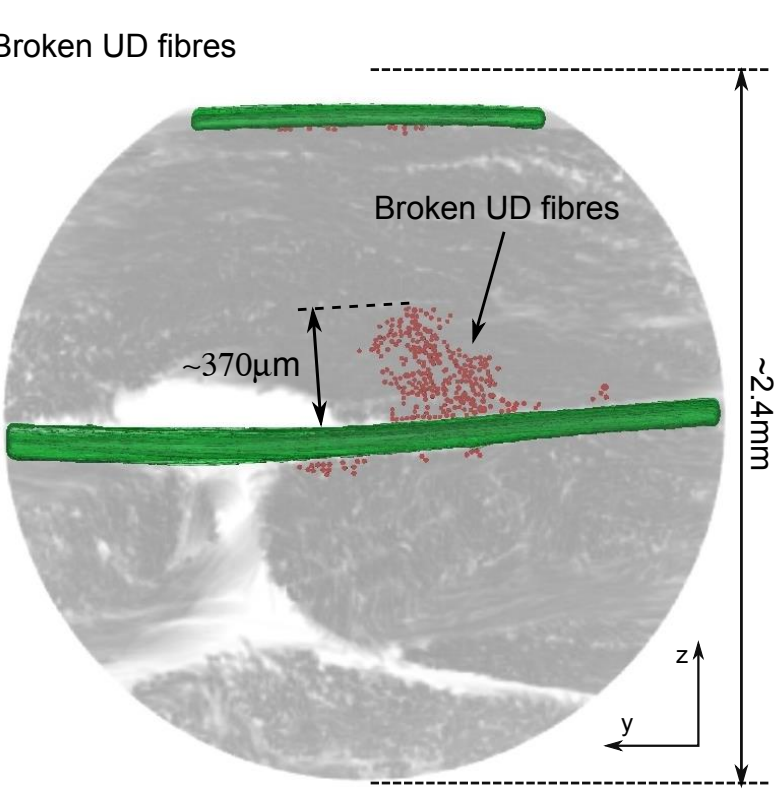

(b) Top view

Figure 14: 3D visualisation and b) top view of fibre fractures for the high resolution scan of S4 (dataset HR4). The fibre fractures are marked as red discs, the thin off-axis backing bundles are marked in green, and the UD fibres have been rendered transparent for clarification.

\section{Discussion of the proposed damage progression scheme}

In the following, the process of damage progression postulated by Zangenberg et al. [11] outlined earlier in section 1.2 will be discussed in the light of the X-ray CT results. From our results it was seen that fibre fractures were only present where the backing bundles intersect and there is no visible distance between the backing and the UD bundles. In the original damage scheme, UD fibre damage was taken to appear mainly at intersecting backing bundles, and in the current study damage was only observed at these intersecting regions. However, in the study by Zangenberg et al.[11], small damage regions were also present at other locations such as near the stitching thread. At this point, it cannot be ruled out that the fibre fractures also exist at these locations 


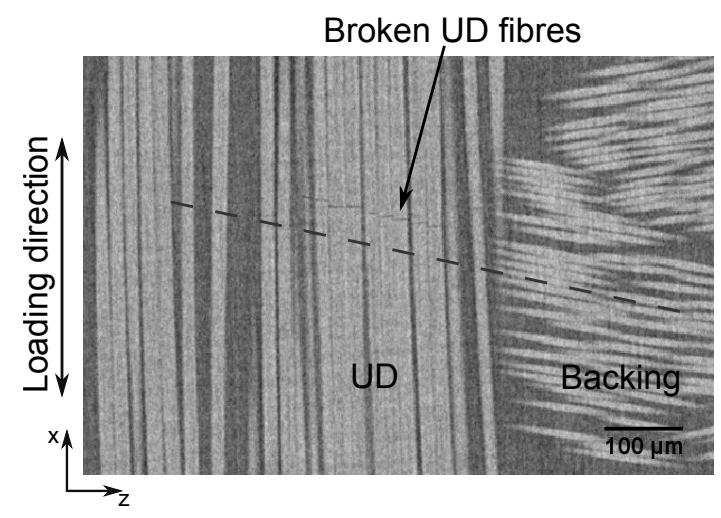

Figure 15: Broken UD fibres just next to the backing for S4. It is seen that the fibre fractures align with the backing fibre orientation.

in the samples considered using CT. That is, it is possible that the crack opening is smaller in these regions, as the damage zone is likely to be smaller. If the opening between fibre fractures is smaller (the cracks are closed), it might not be possible to visualise at $1.2 \mu \mathrm{m}$ voxel size. In addition, the locations of the stitching are difficult to identify, since the stitching thread and matrix have similar densities. This could be investigated by applying tension to the sample during scanning, which is currently on-going work.

During the stage (II) where the stiffness degrades steadily, the damage scheme (Fig. 1) suggests that UD fibre fracture damage zones gradually increase in size as a function of the load cycles. When considering the absolute number of cycles, the size of the observed damage regions is seen to increase with the number of load cycles as shown in Table 4. The penetration depth into the UD bundle is estimated by assuming an average bundle thickness of $0.9 \mathrm{~mm}$. The results in Table 4 support the hypothesis that a gradual loss of stiffness is caused by an increasing number of axial fibre fractures in these local regions, extending further and further into the UD bundles as the original damage scheme states. The damage observed in this study shows that many fractures occur in clusters or chains, which may not be captured by a 2D investigation.

Even though the observations by Zangenberg et al. [11] were obtained at one specific damage level and using destructive 2D visualisation techniques, many of the observa- 
Table 4: Max observed damage depth into UD bundle for different load stages both absolute and normalised with an average UD bundle thickness of $0.9 \mathrm{~mm}$.

\begin{tabular}{llll}
\hline & S2 & S3 & S4 \\
\hline Cycles & 55,000 & 85,000 & 95,000 \\
Penetration depth in UD & $130 \mu \mathrm{m}$ & $310 \mu \mathrm{m}$ & $370 \mu \mathrm{m}$ \\
Penetration depth in UD (\%) & $\sim 14 \%$ & $\sim 34 \%$ & $\sim 41 \%$ \\
\hline
\end{tabular}

tions in this study corroborate their damage scheme. However it is unlikely that damage sites can be reliably identified from 2D imaging. Further, in this study, it is clearly observed that damage progression is a $3 \mathrm{D}$ process, and that looking in $2 \mathrm{D}$ might give misleading results depending on the location of the cut surface. The observed damage zones using X-ray CT comprising single chains of fibre fractures extending in various directions, and even clusters of fibre fractures. Therefore, a 3D view is essential and it is preferable to include the damage progression over time in order to fully understand the damage development. In the future, time-lapse experiments tracking a specific ROI in a sample over the three stages will be reported in order to confirm the growth of the fracture zones. This is currently the focus of ongoing work.

\section{Conclusion}

In this paper, damage evolution under tension fatigue was examined using X-ray computed tomography experiments. A hierarchical approach has been taken using both large field of view medium resolution scanning to identify damage zones and small field of view high resolution scanning to analyse the arrangement of fibre fractures. All the damage sites were located around regions where intersecting backing bundles were located next to the UD bundles with no visible distance in between.

Our results have shown that the fibre fractures, for fibres in contact with the backing, were aligned with the direction of the backing. This suggests that the fibre fractures were initiated by matrix cracks in the backing bundles. In addition, except for just next to the transverse backing bundles, the UD fibre fractures were observed to occur 
as chains and clusters of fibre fractures rather than planes. That is, fibre fractures propagate away from the contacting intersecting backing bundles in chains and planes that could not be determined simply from 2D destructive analyses. In order to properly determine the transition from stage II to stage III, time lapse studies of the fatigue of individual samples are required in the future.

\section{Acknowledgements}

The author would like to acknowledge the assistance provided by the Manchester Xray Imaging Facility, which was funded in part by the EPSRC (grants EP/F007906/1, EP/F001452/1 and EP/I02249X/1). Financial support from CINEMA: "the allianCe

for ImagiNg of Energy MAterials", DSF-grant no. 1305-00032B under "The Danish Council for Strategic Research" is gratefully acknowledged. This research was conducted using mechanical testing equipment from DTU Center for Advanced Structural and Material Testing (CASMAT), Grant No. VKR023193 from Villum Fonden. Additionally we would like to thank LM Wind Power for manufacturing of test specimens.

\section{References}

[1] Global Wind Statistics 2014, http://www.gwec.net/wp-content W/uploads/2015/02/GWEC_GlobalWindStats2014_FINAL_10.2.2015.pdf (2015).

[2] R. P. L. Nijssen, Fatigue life prediction and strength degradation of wind turbine rotor blade composites, PhD Thesis, Delt University of Technology.

[3] P. Brøndsted, R. P. L. Nijssen, Fatigue as a design driver for composite wind turbine blades, Advances in Wind Turbine Blade Design and Materials (2013) 175-209doi:10.1533/9780857097286.2.175.

[4] A. Gagel, D. Lange, K. Schulte, On the relation between crack densities, stiffness degradation, and surface temperature distribution of tensile fatigue loaded glassfibre non-crimp-fabric reinforced epoxy, Composites Part A - Applied Science and Manufacturing 37 (2) (2006) 222-228. doi:10.1016/j.compositesa.2005.03.028. 
[5] F. Edgren, D. Mattsson, L. E. Asp, J. Varna, Formation of damage and its effects on non-crimp fabric reinforced composites loaded in tension, Composites Science and Technology 64 (5) (2004) 675-692. doi:10.1016/s0266-3538(03)00292-6.

[6] J. Tong, F. J. Guild, S. L. Orgin, P. A. Smith, On matrix crack growth in quasiisotropic laminates - I. experimental investigation, Composites Science and Technology 57 (11) (1997) 1527-1535. doi:10.1016/S0266-3538(97)00080-8.

[7] J. Varna, R. Joffe, N. V. Akshantala, R. Talreja, Damage in composite laminates with off-axis plies, Composites Science and Technology 59 (1999) 2139-2147. doi:10.1016/S0266-3538(99)00070-6.

[8] M. Quaresimin, P. A. Carraro, L. P. Mikkelsen, N. Lucato, L. Vivian, P. Brøndsted, B. F. Sørensen, J. Varna, R. Talreja, Damage evolution under cyclic multiaxial stress state: A comparative analysis between glass/epoxy laminates and tubes, Composites: Part B 61 (2014) 282-290. doi:10.1016/j.compositesb.2014.01.056.

[9] K. L. Reifsnider, R. Jamison, Fracture of fatigue-loaded composite laminates, International Journal of Fatigue 4 (4) (1982) 187-197. doi:10.1016/0142-1123(82)900019 .

[10] R. Jamison, K. Schulte, K. Reifsnider, W. Stinchcomb, Characterization and Analysis of Damage Mechanisms in Tension-Tension Fatigue of Graphite/Epoxy Laminates, Effects of Defects in Composite Materials (1984) 2121-35doi:10.1520/STP30196S.

[11] J. Zangenberg, P. Brøndsted, J. W. Gillespie Jr., Fatigue damage propagation in unidirectional glass fibre reinforced composites made of a non-crimp fabric, Journal of Composite Materials 48 (22) (2014) 2711-2727. doi:10.1177/0021998313502062.

[12] F. Sket, R. Seltzer, J. M. Molina-Aldareguía, C. Gonzalez, J. LLorca, Determination of damage micromechanisms and fracture resistance of glass fiber/epoxy cross- 
ply laminate by means of X-ray computed microtomography, Composites Science and Technology 72 (2) (2012) 350-359. doi:10.1016/j.compscitech.2011.11.025.

[13] S. R. Stock, X-ray microtomography of materials, International Materials Reviews 44 (4) (1999) 141-164.

[14] E. Maire, P. J. Withers, Quantitative X-ray tomography, International Materials Reviews 59 (1) (2014) 1-43. doi:10.1179/1743280413y.0000000023.

[15] P. J. Withers, M. Preuss, Fatigue and damage in structural materials studied by X-Ray tomography, Annual Review of Materials Research 42 (2012) 81-103. doi:10.1146/annurev-matsci-070511-155111.

[16] P. Wright, X. Fu, I. Sinclair, S. M. Spearing, Ultra high resolution computed tomography of damage in notched carbon fiber-epoxy composites, Journal of Composite Materials 42 (19) (2008) 1993-2002. doi:10.1177/0021998308092211.

[17] P. Wright, A. Moffat, I. Sinclair, S. M. Spearing, High resolution tomographic imaging and modelling of notch tip damage in a laminated composite, Composites Science and Technology 70 (10) (2010) 1444-1452. doi:10.1016/j.compscitech.2010.04.012.

[18] A. E. Scott, M. Mavrigirdati, P. Wright, I. Sinclair, S. M. Spearing, In situ fibre fracture measurement in carbon-epoxy laminates using high resolution computed tomography, Composites Science and Technology 71 (12) (2011) 1471-1477. doi:10.1016/j.compscitech.2011.06.004.

[19] S. C. Garcea, M. Macrigirdato, A. E. Scott, I. Sinclair, S. M. Spearing, Fatigue micromechanism characterisation in carbon fibre reinforced polymers using synchrotron radiation computed tomography, Composites Science and Technology 99 (88) (2014) 23-30. doi:10.1016/j.compscitech.2014.05.006.

[20] S. C. Garcea, I. Sinclair, S. M. Spearing, In situ synchrotron tomographic evaluation of the effect of toughening strategies on fatigue micromechanisms in carbon 
fibre reinforced polymers, Composites Science and Technology 109 (2015) 32-39. doi:10.1016/j.compscitech.2015.01.012.

[21] B. M. Patterson, N. L. Cordes, K. Henderson, J. C. E. Mertens, A. J. Clarke, B. Hornberger, A. Merkle, S. Etchin, A. Tkachuk, M. Leibowitz, D. Trapp, W. Qiu, B. Zhang, H. Bale, X. Lu, R. Hartwell, P. J. Withers, R. S. Bradley, In Situ Laboratory-Based Transmission X-Ray Microscopy and Tomography of Material Deformation at the Nanoscale, Experimental Mechanics (2016) 113doi:10.1007/s11340-016-0197-3.

[22] P. J. Schilling, B. P. R. Karedla, A. K. Tatiparthi, M. A. Verges, P. D. Herrington, X-ray computed microtomography of internal damage in fiber reinforced polymer matrix composites, Composites Science and Technology 65 (14) (2005) 2071-2078. doi:10.1016/j.compscitech.2005.05.014.

[23] B. Yu, R. Blanc, C. Soutis, W. P. J., Evolution of damage during the fatigue of 3D woven glass-fibre reinforced composites subjected to tension-tension loading observed by time-lapse X-ray tomography, Composites: Part A 82 (2016) 279-290. doi:10.1016/j.compositesa.2015.09.001.

[24] B. Yu, R. S. Bradley, C. Soutis, P. J. Hogg, P. J. Withers, 2D and 3D imaging of fatigue failure mechanisms of 3D woven composites, Composites Science and Technology 77 (2015) 37-49. doi:10.1016/j.compositesa.2015.06.013.

[25] S. Topal, L. Baiocchi, A. D. Crocombe, S. L. Ogin, P. Potluri, P. J. Withers, M. Quaresimin, P. A. Smith, M. C. Poole, A. E. Bogdanovich, Late-stage fatigue damage in a 3D orthogonal non-crimp woven composite: An experimental and numerical study, Composites Part A: Applied Science and Manufacturing 79 (2015) 155-163. doi:10.1016/j.compositesa.2015.08.020.

URL http://dx.doi.org/10.1016/j.compositesa.2015.08.020 
[26] J. Zangenberg, The effects of fibre architecture on fatigue life-time of composite materials, PhD Thesis, DTU Wind Energy PhD-0018(EN).

[27] ISO, Plastics - Determination of tensile properties - Part 5: Test conditions for unidirectional fibre-reinforced plastic composites, ISO 527-5:2009(E), International Organization for Standardization, Geneva, Switzerland (2009).

[28] R. Talreja, Fatigue of composite materials: damage mechanisms and fatigue-life diagrams, Proc. R. Soc. Lond. A 378 (1981) 461-475. doi:10.1098/rspa.1983.0054.

[29] J. Banhart, Advanced Tomographic Methods in Materials Research and Engineering, Oxford Univ. Press, 2008. doi:10.1093/acprof:oso/9780199213245.001.0001.

[30] K. M. Jespersen, J. Zangenberg, T. Lowe, P. Withers, L. Mikkelsen, X-ray CT data: fatigue damage in glass fibre/polyester composite used for wind turbine blades [Data set], http://doi.org/10.5281/zenodo.154714 (2016).

[31] K. M. Jespersen, J. Zangenberg, L. P. Mikkelsen, Micromechanical investigation of fatigue damage in uni-directional fibre composites, in: Proceedings of 6th International Conference on Fatigue of Composites, 2015, http://orbit.dtu.dk/files/108663074/ICFCPaperDraft_KMUN_Final.pdf.

[32] S. F. Shuler, J. W. Holmes, X. Wu, D. Roach, Influence of Loading Frequency on the Room-Temperature Fatigue of a Carbon-Fiber/SiC-Matrix Composite, Journal of the American Ceramic Society 76 (9) (1993) 2327-2336. doi:10.1111/j.11512916.1993.tb07772.x.

[33] N. Chawla, Y. Tur, J. Holmes, J. Barber, A. Szweda, High-frequency fatigue behavior of woven-fiber-fabric-reinforced polymer-derived ceramic-matrix composites, Journal of the American Ceramic Society 81 (5) (1998) 1221-1230.

[34] K. M. Jespersen, T. Lowe, P. Withers, J. Zangenberg, L. Mikkelsen, Micromechanical time-lapse X-ray CT study of fatigue damage in uni-directional fibre 
composites, in: 20th International Conference on Composite Materials, 2015, http://iccm20.org/fullpapers/file?f=nDdmY09mvY. 